\title{
Pneumococcal vaccination and efficacy in patients with heterotaxy syndrome
}

\author{
Pei-Lan Shao ${ }^{1}$, Mei-Hwan $\mathrm{Wu}^{2}$, Jou-Kou Wang ${ }^{2}$, Hui-Wen Hsu², Li-Min Huang ${ }^{2}$ and Shuenn-Nan Chiu ${ }^{2}$
}

BACKGROUND: Pneumococcal vaccines, including pneumococcal polysaccharide vaccine (PPV) and pneumococcal conjugated vaccine (PCV), are crucial in preventing invasive pneumococcal diseases. We analyzed the pneumococcal vaccination rate, efficacy, and durability in patients with heterotaxy.

METHODS: All patients with heterotaxy and CCHD who were followed up at our institution between 2010 and 2015 were included. Pneumococcal vaccine status and geometric mean concentration (GMC) of serotypes 6B, 14, 19F, and 23F were analyzed. Splenic function was considered abnormal when the percentage of IgM memory B cell was less than $1 \%$.

RESULTS: The GMCs of the four serotypes did not differ significantly between patients with heterotaxy and those with CCHD; the GMCs were also not affected by abnormal splenic function. Most patients had GMCs $>0.35 \mu \mathrm{g} / \mathrm{ml}$ (protection level) 4-5 years after either PPV or PCV injection; however, it may decay gradually in some serotypes. In addition, $21.4 \%$ of 42 patients with heterotaxy did not receive pneumococcal vaccine, and none completely adhered to the vaccine guidelines.

CONCLUSIONS: Vaccine efficacy was acceptable, even in patients with abnormal splenic function. In some patients, the durability of PPV and PCV decreased with time, highlighting the importance of booster doses. Vaccination rate in patients with heterotaxy is unsatisfactory.

$\mathbf{H}$ eterotaxy syndrome is a developmental defect that involves abnormal arrangement of the left-right axis of the thoracic and visceral organs. Because of abnormal cardiac looping, complex congenital heart disease (CCHD) is common in patients with heterotaxy, and multiple-stage operation (i.e., a Fontan-type operation) is necessary for their long-term survival $(1,2)$. In addition, spleen agenesis or dysgenesis is common in these patients, making them susceptible to fulminant encapsulated bacterial infection (particularly, pneumococcus) $(3,4)$. In our previous study, the 5-year community-acquired sepsis rate was $14.5 \%$ and the 5 -year all-cause mortality rate was $48 \%$ in patients with heterotaxy (5). Community-acquired and postoperative infection-related mortality accounted for $40 \%$ of all deaths (5). Antibiotics prophylaxis and vaccination are crucial in preventing infections in these high-risk patients (6-8).

Pneumococcal infection is one of the most common causes of bacterial infection in infants and children $(9,10)$. In patients with heterotaxy, invasive pneumococcal disease and related fulminant sepsis are even more common $(5,11)$. With the introduction of pneumococcal vaccine, mortality caused by pneumococcal infection has decreased significantly $(10,12)$. The National Health Insurance Administration in our country has been reimbursing pneumococcal conjugated vaccine (PCV) prophylaxis in patients with certain congenital heart diseases (e.g., heterotaxy syndrome and CCHD) since July 2009. The dosing schedule of PCVs is $3+1$ doses in children younger than 6 months, $2+1$ doses in those aged between 6 months and 1 year, and 2 doses in those older than 1 year. According to the immunization guidelines for asplenism and splenic hypofunction, hyposplenic children aged 2-5 years should receive one dose of pneumococcal polysaccharide vaccine (PPV) if they are previously fully immunized with PCV13; one dose of PCV13, followed by PPV 2 months later, if they are previously immunized with PCV7; and two doses of PCV13 2 months apart, followed by PPV 2 months later, if they are not previously immunized with pneumococcal vaccine $(8,13)$. In addition, a PPV booster is necessary after 5 years (13). In this study, using a cohort of patients with heterotaxy and CCHD who were followed up at our center, we investigated their adherence to the pneumococcal vaccine guidelines and analyzed the efficacy and durability of the vaccine in these patients.

\section{METHODS}

\section{Subjects}

The study cohort comprised all patients older than 1 year who had been diagnosed with heterotaxy syndrome or CCHD and were followed up between January 2010 and December 2015 at the National Taiwan University Hospital, the referral center for heterotaxy syndrome and CCHD in Taiwan. The diagnostic criteria for heterotaxy syndrome included bilateral atrial appendage morphology belonging to either the right atrium or the left atrium accompanied by bilateral symmetric tracheobronchial trees, confirmed during surgery or through computed tomography imaging. We defined CCHD as CHD that required single ventricle physiology

${ }^{1}$ Department of Laboratory Medicine and Department of Pediatrics, National Taiwan University Hospital Hsin-Chu Branch, Hsinchu, Taiwan; ${ }^{2}$ Department of Pediatrics, National Taiwan University Hospital and Medical College, Taipei, Taiwan. Correspondence: Shuenn-Nan Chiu (michael@ntuh.gov.tw)

Received 19 June 2016; accepted 23 January 2017; advance online publication 24 May 2017. doi:10.1038/pr.2017.39 
surgery; this included tricuspid atresia (eight patients), double inlet left ventricle (eight patients), hypoplastic left heart syndrome (seven patients), double outlet right ventricle (six patients), l-transposition of the great arteries (six patients), double inlet double outlet ventricle (eight patients), mitral atresia with double outlet right ventricle (four patients), pulmonary atresia with intact ventricular septum (three patients), and others (four patients).

We conducted our study in accordance with the regulations of the Institutional Review Board of the National Taiwan University Hospital, which in turn abides by the Declaration of Helsinki. After receiving written informed consent from the patients or their parents, we reviewed patient charts and recorded their complete vaccination history. The patients completed a questionnaire on relevant prior vaccinations. In addition, we reviewed the medical records for a history of admissions because of community-acquired pneumonia, sepsis, and nosocomial sepsis.

\section{Pneumococcal Antibody Assay}

In the second part of the study, we investigated the GMCs of serotypes $6 \mathrm{~B}, 14,19 \mathrm{~F}$, and $23 \mathrm{~F}$ in these patients. Five milliliters of fresh blood was sampled from peripheral blood or during cardiac catheterization and stored at $-80^{\circ} \mathrm{C}$. Levels of anticapsular antibody were determined using enzyme-linked immunoassay (ELISA) by following methods described previously but with minor modifications (14). Briefly, the sample and positive control were double-adsorbed with cell wall polysaccharide and incubated at room temperature for $30 \mathrm{~min}$. Capsular polysaccharides from pneumococcus serotypes 6B, 14, 19F, or $23 \mathrm{~F}$ obtained from the American Type Culture Collection were suspended in phosphate-buffered saline ( $\mathrm{pH} 7.4)$ at a concentration of $10 \mu \mathrm{g} / \mathrm{ml}$ and were used directly to coat wells through overnight incubation at $4{ }^{\circ} \mathrm{C}$. After washing, blocking was performed overnight at $4{ }^{\circ} \mathrm{C}$ with phosphate-buffered saline containing $1 \%$ bovine serum albumin. Duplicate serum samples were studied in twofold serial dilutions, and human antipneumococcal capsule reference serum (first International Standard of the World Health Organization International Standard; National Institute for Biological Standards and Control code: $007 \mathrm{sp}$ ) was included in each plate as a positive control at room temperature for $2 \mathrm{~h}$. After washing, the first antibody incubation was performed at room temperature for $2 \mathrm{~h}$. The wells were thoroughly washed to remove unbound antibodies, and horseradishperoxidase-conjugated goat antibody to $1 \mathrm{mg} / \mathrm{ml}$ human IgG (Novex by Life Technologies, Carlsbad, CA) at a 1:5,500 dilution was used to detect IgG. The reaction occurred at $10 \mathrm{~min}$ in the dark by addition of TMB substrate (Becton Dickinson, Franklin Lakes, NJ); this was followed by the addition of $1 \mathrm{~N}$ sulfuric acid to stop the reaction. All washings between incubation were performed with phosphatebuffered saline buffer containing $0.05 \%$ Tween 20 . Optical density was read using an ELISA reader (VersaMax ELISA Microplate Reader, Molecular Devices, Sunnyvale, CA) at $450 \mathrm{~nm}$.

\section{Splenic Function Assessment: Flow Cytometry Assay for Detection of IgM Memory B-cell Percentage}

Mononuclear cells were isolated from heparinized blood by using Ficoll-Paque Plus (GE Healthcare Bio-Sciences, Chicago, IL) and stained with the appropriate antibody combinations of fluorescein isothiocyanate, phycoerythrin, peridinin-chlorophyll protein complex, and allophycocyanin. Monoclonal antibodies HIB19 (antiCD19), HIB22 (anti-CD22), M-T271 (anti-CD27), G20-127 (antiIgM), and IA6-2 (anti-IgD) were obtained from Becton Dickinson. Dead cells were excluded from analysis by using forward- and sidescatter gating. All analyses were performed through four-color flow cytometry (FACSCalibur; Becton Dickinson) interfaced with the Macintosh CellQuest Computer Program. The CD19+ cells, identified as B lymphocytes, were gated for subsequent analysis. The percentage of IgM memory B cells was calculated according to the percentage of immunophenotyping of the surface markers of the $\mathrm{CD} 22+, \mathrm{CD} 27+, \mathrm{IgM}+$, and IgD+ cells in the CD22+ cells. According to previous reports, IgM memory B-cell percentages less than $1 \%$ were considered low $(15,16)$.
Table 1. Basic clinical characteristics and vaccination status between patients with heterotaxy and CCHD

\begin{tabular}{lcc}
\hline & $\begin{array}{c}\text { Heterotaxy } \\
(\boldsymbol{n}=\mathbf{4 2})\end{array}$ & $\begin{array}{c}\text { CCHD } \\
(\boldsymbol{n}=\mathbf{5 4})\end{array}$ \\
\hline Age & $7.5 \pm 5.1$ & $6.4 \pm 4.5$ \\
Gender (male, \%) & $25(59.5 \%)$ & $27(50 \%)$ \\
No spleen by image* & $27(64.3 \%)$ & 0 \\
Memory B cell (\%) ${ }^{\mathrm{a},{ }^{*}}$ & $8.0 \pm 6.4$ & $13.7 \pm 12.1$ \\
IgM memory B cell (\%) ${ }^{\mathrm{a},{ }^{*}}$ & $1.9 \pm 2.2$ & $4.2 \pm 3.8$ \\
Low IgM memory B cell (<1\%) ${ }^{\mathrm{a}, * *}$ & $11(30.6 \%)$ & $6(12.0 \%)$ \\
Community-acquired sepsis & $3(7.1 \%)$ & 0 \\
Community-acquired pneumonia & $6(14.3 \%)$ & $6(11.3 \%)$ \\
Nosocomial sepsis & $9(22 \%)$ & $15(28.8 \%)$
\end{tabular}

\begin{tabular}{|c|c|c|}
\hline \multicolumn{3}{|l|}{ PCV vaccine } \\
\hline Number injected & $26(61.9 \%)$ & $29(53.7 \%)$ \\
\hline PCV10 or PCV13 booster (no, \%) & $3(12.5 \%)$ & 0 \\
\hline PCV vaccine dose & $2.9 \pm 1.2$ & $2.5 \pm 1.0$ \\
\hline Mean age of last vaccine & $2.3 \pm 1.6$ & $2.0 \pm 1.1$ \\
\hline $\begin{array}{l}\text { Duration between antibody titers and } \\
\text { last vaccine (years) }\end{array}$ & $2.8 \pm 1.5$ & $2.5 \pm 1.6$ \\
\hline PPV vaccine number injected & $9(21.4 \%)$ & $5(9.3 \%)$ \\
\hline Mean age of last vaccine & $3.6 \pm 2.4$ & $4.4 \pm 2.9$ \\
\hline $\begin{array}{l}\text { Duration between antibody titers and } \\
\text { last vaccine (years) }\end{array}$ & $9.9 \pm 2.4$ & $6.9 \pm 3.9$ \\
\hline \multicolumn{3}{|c|}{$\begin{array}{l}\text { CCHD, complex congenital heart disease; } P C V \text {, pneumococcal conjugated vaccine } \\
\text { PPV, pneumococcal polysaccharide vaccine. } \\
{ }^{*} P \text { value }<0.01 ;{ }^{* *} P \text { value }<0.05 \text {. } \\
\text { a Only } 36 \text { patients with heterotaxy syndrome and } 50 \text { patients with } C C H D \text { received } \\
\text { IgM memory B-cell test. } \\
\text { bThe number and percentage of patients who received either PCV10 or PCV13 } \\
\text { booster after previous PCV7 injection. }\end{array}$} \\
\hline
\end{tabular}

\section{Statistical Analysis}

SPSS version 15.0 (SPSS, Chicago, IL) was used to perform statistical analysis. We used Student's $t$-test or one-way analysis of variance to compare the data with normal distribution and a nonparametric method with the Mann-Whitney or Kruskal-Wallis test to compare the data with skewed distribution. Categorical data were examined using the $\chi^{2}$-test and Fisher's exact test. The data were presented as mean \pm s.d.; $P<0.05$ was considered statistically significant.

\section{RESULTS}

The basic demographic data of the 42 patients with heterotaxy and 54 patients with CCHD are listed in Table 1. In the heterotaxy group, 38 had right atrial isomerism and 4 had left atrial isomerism. Imaging studies could not detect the spleen in $64.3 \%$ of patients with heterotaxy. Community-acquired sepsis or severe bacterial infection occurred in three $(7.1 \%)$ patients with heterotaxy. One of the three patients acquired pneumococcal meningitis through a nonvaccine strain (15B), although he had received four doses of PCV7. The other two patients acquired pneumococcal sepsis and brain abscess with unknown pathogen, respectively, without previous pneumococcal vaccine prophylaxis. The nosocomial sepsis rate was similar in patients with heterotaxy $(22 \%)$ and those with 


\section{Pneumococcal vaccination in heterotaxy $\mid$ Articles}
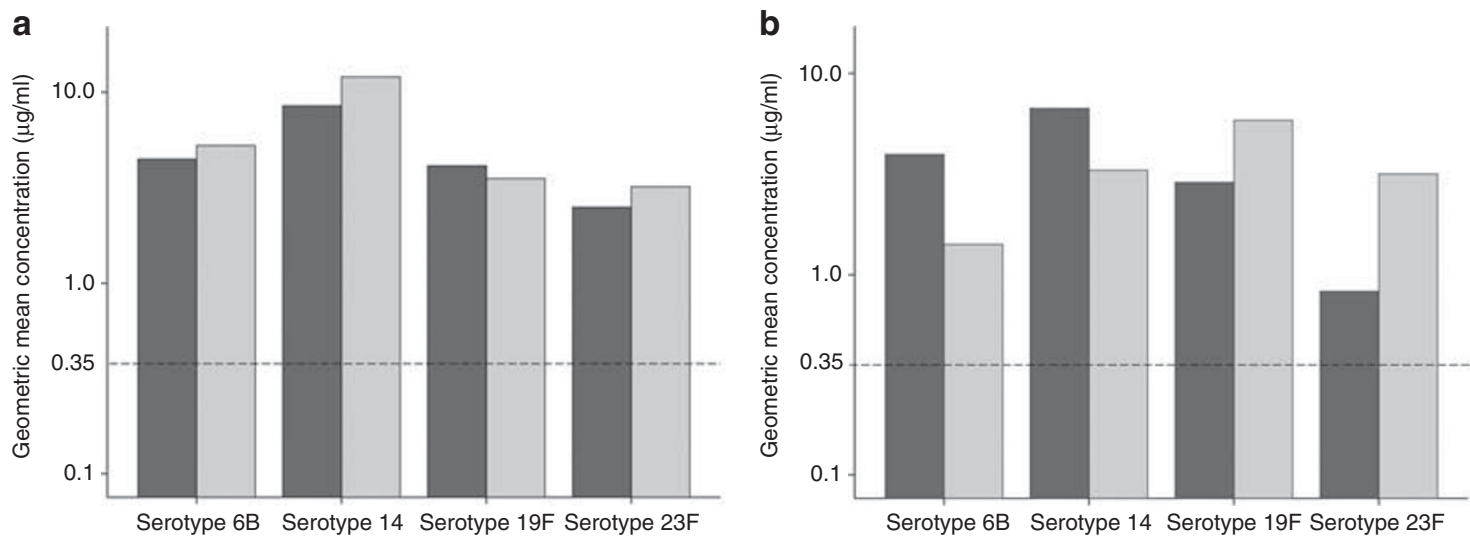

Figure 1. Comparisons of the geometric mean concentrations (GMCs) in four serotypes (6B, 14, 19F, and 23F) between patients with heterotaxy syndrome (closed bar) and complex congenital heart disease (CCHD, open bar) after vaccination with pneumococcal conjugated vaccine (PCV; a) and pneumococcal polysaccharide vaccine (PPV; $\mathbf{b})$. We could see that the GMCs of four serotypes showed no significant differences between these two groups after either PCV or PPV injection.

CCHD (28.8\%). For the splenic immunological function, the percentages of memory B cell and IgM memory B cell were significantly lower in the heterotaxy group compared with the CCHD group. Low percentage of IgM memory B cells $(<1 \%)$ was found in $30.6 \%$ of patients with heterotaxy and in only $12 \%$ of patients with CCHD $(P=0.053)$.

\section{Pneumococcal Vaccination}

In our cohort, $61.9 \%$ of patients with heterotaxy and $53.7 \%$ of patients with CCHD received PCVs, and $21.4 \%$ of patients with heterotaxy and $9.3 \%$ of patients with CCHD received PPV. Therefore, $21.4 \%$ of patients with heterotaxy and $37 \%$ of patients with CCHD had not received pneumococcal vaccine prophylaxis. However, in recent years, adherence to pneumococcal vaccine prophylaxis has improved. The vaccination rate increased from $51.3 \%$ for patients born before 2005 to $82.5 \%$ for those born after 2005 .

Of the 42 patients with heterotaxy, none adhered completely to the guidelines. Of the 24 patients who received PCV7 initially, only 3 (12.5\%) received PCV10 or PCV13 booster later. Of the 26 patients who received PCVs, none received additional PPV booster. No significant difference was found between the heterotaxy and CCHD groups in the age at which patients received last pneumococcal vaccine, either PCV or PPV.

\section{Efficacy of Pneumococcal Vaccine}

Figure 1 presents a comparison between the heterotaxy and CCHD groups of the geometric mean concentrations (GMCs) of serotypes $6 \mathrm{~B}, 14,19 \mathrm{~F}$, and $23 \mathrm{~F}$ in patients receiving PCV and PPV. The mean ages of patients in the heterotaxy and CCHD groups were 7.5 and 6.4 years, respectively. Antibody titers showed no significant differences in these four serotypes between the heterotaxy and CCHD groups, after either PCV or PPV injections.

In the heterotaxy group, we determined whether abnormal splenic function, defined as a low percentage of IgM memory
B cells, would affect vaccine efficacy (Figure 2). The antibody titer revealed no significant differences between those with normal and abnormal splenic functions (Figure $3 a$ ). Moreover, we investigated whether the presence of spleen, defined through an imaging study, would interfere with vaccine efficacy (Figure $3 \boldsymbol{b}$ ). Again, the antibody titers revealed no significant differences between those with and those without the spleen.

\section{Durability of Vaccine Effect}

We determined the durability of the pneumococcal vaccine in patients with heterotaxy and CCHD, and found that GMCs in the four serotypes were persistently high $4-5$ years after PCV injection (Figure 4). This result suggests that the protection effect of PCV lasts for at least 4-5 years. For PPV injection, the titers of pneumococcal antibody were persistently high more than 5 years after vaccination in all four serotypes.

Because serotype-specific GMC of $0.35 \mu \mathrm{g} / \mathrm{ml}$ or higher has been reported to predict protection against invasive pneumococcal diseases $(17,18)$, we investigated the proportion of low GMC in patients with heterotaxy and CCHD. In total, 4 of the 54 patients who received PCV injection had low GMC $(<0.35 \mu \mathrm{g} / \mathrm{ml})$, and 2 of the 15 patients who received PPV injection had low GMC. The low GMC was found in one patient for serotype $6 \mathrm{~B}$ and in the other five patients for serotype 23F. Two of these patients belonged to the heterotaxy group and the other four belonged to the CCHD group. Splenic function, spleen status, PCV vs. PPV, and PCV doses did not differ significantly between those with low GMCs and those with normal GMCs. However, patients with low GMC had longer duration between their last vaccination and antibody titer check $(6.3 \pm 3.6$ vs. $3.5 \pm 2.9$ years, $P=0.031)$.

\section{DISCUSSION}

In the present study, we identified that (i) adherence to the pneumococcal vaccine guidelines in patients with heterotaxy is poor. Although the National Health Insurance Administration 

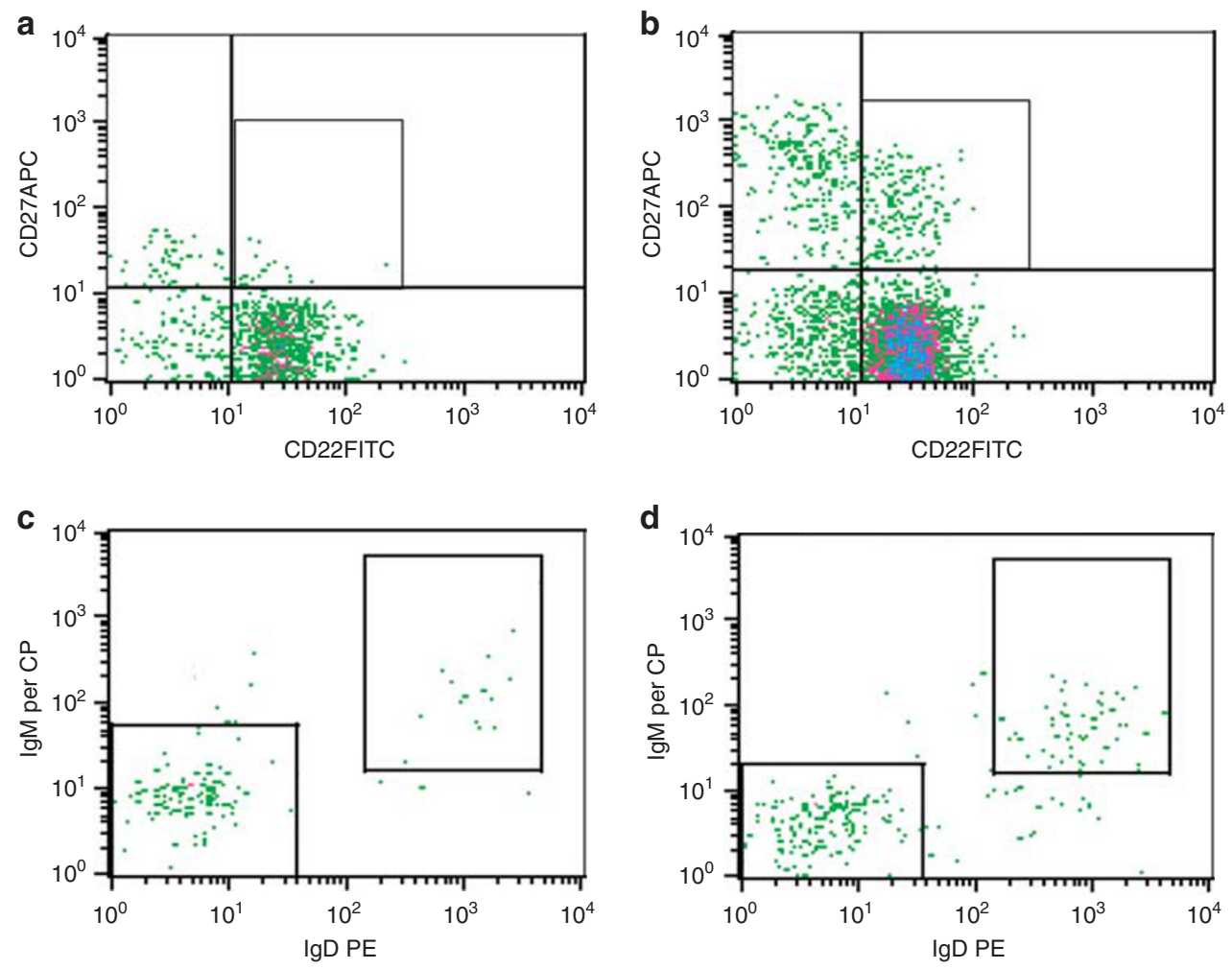

Figure 2. Flow cytometry analysis of IgM memory B cells in patients of right atrial isomerism with and without previous bacterial infections. Upper panel is the peripheral blood mononuclear cells stained with antibodies to CD19, CD22, and CD27 by using three-color flow cytometry. Data are illustrated as a density plot. The memory B cells $\left(C D 22^{+} 27^{+}\right)$are denoted as rectangles. Lower panel is the peripheral blood mononuclear cells stained with antibodies to CD22, CD27, IgM, and IgD, and analyzed using four-color flow cytometry. The memory $B$ cells $\left(C D 22^{+} C D 27^{+}\right)$were gated for further analysis. The upper-right rectangles illustrate $\lg M$ memory $B$ cells $\left(\lg M^{+} \lg D^{+}\right)$. (a,c) A 5.9-year-old boy with right atrial isomerism, who had previous pneumococcal meningitis (15B) at the age of 5 years, had low memory B cells (1.1\%) and lgM memory B cells $(0.1 \%)$. He received four doses of pneumococcal conjugated vaccine 3.9 years ago, and the geometric mean concentrations (GMCs) of the four serotypes (6B, 14, 19F, and 23F) were all above $1 \mu \mathrm{g} / \mathrm{ml}$. (b,d) A 6.3-year-old boy with right atrial isomerism, who had no previous severe bacterial infection, had low memory $B$ cell (6.3\%) but normal IgM memory B cell (1.3\%). He received two doses of pneumococcal conjugated vaccine 3.9 years ago, and the GMCs of four serotypes were also above $1 \mu \mathrm{g} / \mathrm{ml}$. APC, allophycocyanin; FITC, fluorescein isothiocyanate; PE, phycoerythrin; per CP- peridinin chlorophyll protein complex.

a

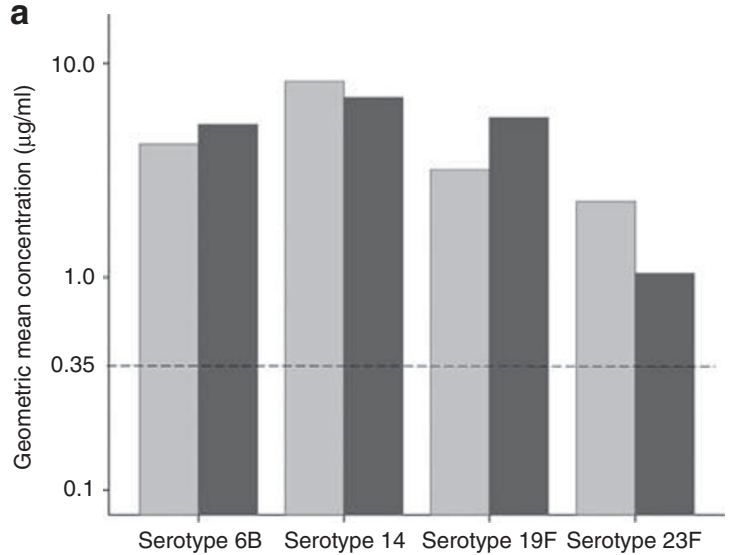

b

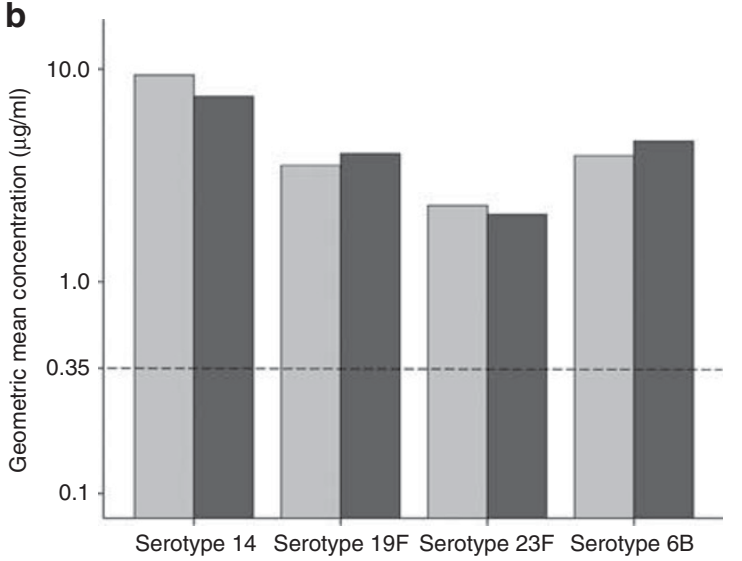

Figure 3. Geometric mean concentrations according to splenic function. (a) Comparisons of the geometric mean concentrations (GMCs) between those with low (closed bar) and normal (open bar) IgM memory B cell. No significant difference was found between the two groups. (b) Comparisons of the GMCs between those with spleen found at imaging study (open bar) and those without (closed bar). Again, no significant difference was found between the two groups. 


\section{Pneumococcal vaccination in heterotaxy $\quad$ Articles}

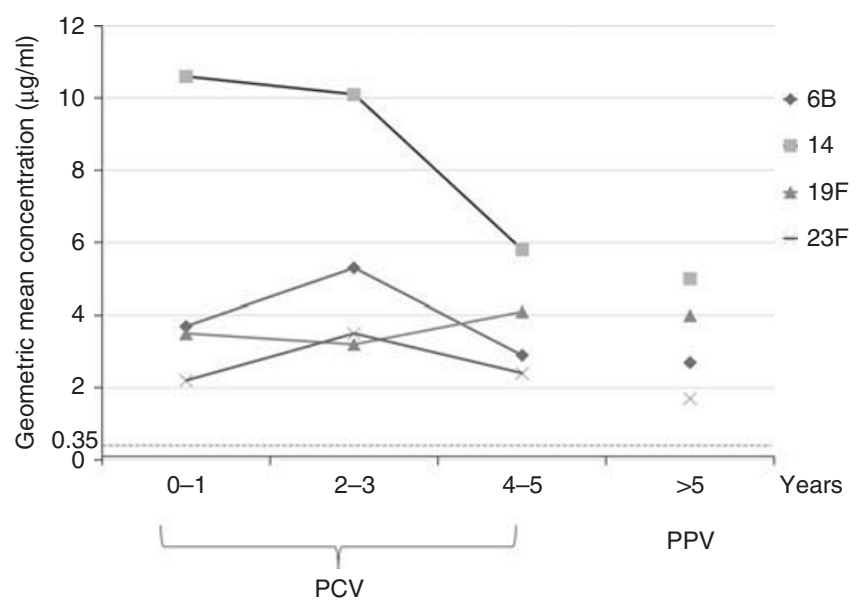

Figure 4. The relationship of geometric mean concentrations (GMCs) in four serotypes $(6 \mathrm{~B}, 14,19 \mathrm{~F}$, and $23 \mathrm{~F})$ with time after pneumococcal conjugated vaccine (PCV) and pneumococcal polysaccharide vaccine (PPV) injections. The mean GMCs of the four serotypes are consistently high for those receiving PCV within 5 years. For those receiving PPV, after more than 5 years, the GMCs of all the four serotypes were still above the protection level.

reimburses PCV prophylaxis for patients with heterotaxy, onefifth of the patients did not receive a pneumococcal vaccine. (ii) The serotype-specific GMCs did not differ significantly between patients with heterotaxy and those with CCHD, meaning that the efficacy of pneumococcal vaccine was equal in both groups. Furthermore, abnormal splenic function did not interfere with the serotype-specific GMCs. (iii) The PCVs and PPVs were efficacious for at least $4-5$ and $>5$ years, respectively, in most patients. However, the GMCs of certain serotypes decreased with time and may be lower than the protection level in a small group of patients.

Pneumococcal infection is one of the most common infectious diseases in humans (9). The risk of invasive pneumococcal disease is especially high during the first 2 years of age. According to the World Health Organization, pneumococcus has accounted for $\sim 1$ million deaths worldwide in children $<5$ years ( (ref. 9)). The introduction of PPV in 1977 reduced the severity of pneumococcal infection in elderly patients (19). Because PPV induces immunogenicity through a T-cell-independent pathway, the response is poor and the protection effect is low in children $<2$ years ( (ref. 20)). A new pneumococcal vaccine (i.e., PCV), a conjugated polysaccharide with a protein carrier, solved this problem. The introduction of PCV7 in 2000 reduced the incidence of invasive pneumococcal disease by $69-89 \%$ in the United States, especially in young children $(9,21,22)$. The introduction of PCV7 has reduced the incidence of invasive pneumococcal disease by $58 \%$ in the pediatric population in Taiwan (23). Serotype replacement became a problem after the introduction of PCV7, and PCV13 booster has been recommended to provide broader coverage $(9,12,20)$. Nevertheless, the introduction of pneumococcal vaccine has profoundly changed the epidemiology of pneumococcal disease.
Adherence to the Pneumococcal Vaccine Guidelines in Patients with Heterotaxy

Patients with heterotaxy have an especially high risk of pneumococcal infection because of dysgenesis or agenesis of the spleen (3-5). Even after treatment with appropriate antimicrobial agents, the mortality rate is high (5). The guidelines of the Advisory Committee on Immunization Practices for immunization in asplenism and splenic hypofunction recommend that pneumococcal vaccination be given to all patients with heterotaxy $(8,13)$. In Taiwan, the National Health Insurance Administration reimburses the costs for pneumococcal vaccine in patients with heterotaxy and CCHD. However, we found that one-fifth of the patients with heterotaxy had received neither PCV nor PPV prophylaxis. Besides, none had completely adhered to the aforementioned guidelines. Nevertheless, the vaccine administration rate among these high-risk patients has increased in recent years after the National Health Insurance Administration began reimbursing the costs of pneumococcal vaccines. Currently, more than $80 \%$ of patients with heterotaxy or CCHD receive a pneumococcal vaccine.

The serotype shift, which refers to the reduction of vaccine strains and increase in nonvaccine strains, is an emerging problem (9). Serotype 19A, which is notorious for its antibiotic resistance and virulence, has become the major serotype for invasive pneumococcal diseases in recent years $(23,24)$, highlighting the importance of booster vaccines (e.g., PCV10 and PCV13). However, we found that the booster rate was low: only $12 \%$ of the patients with heterotaxy who had previously received PCV7 had received PCV10 or 13 boosters. In addition, none of the patients in our cohort had received additional PPV boosters. We interviewed 10 of the attending pediatric cardiologists in our institution and found that none were familiar with the new vaccination guidelines for heterotaxy syndrome. Because most of the patients of heterotaxy with congenital heart disease were followed up at pediatric cardiology clinics, providing further education to the health-care professionals is important to improve the adherence to the pneumococcal vaccination guideline.

\section{Efficacy and Durability of Pneumococcal Vaccines}

In normal children $>2$ years of age, both PPV and PCV can induce satisfactory immune responses. In the study by Sigurdardottir et al, (25) the GMCs in most serotypes can achieve protective level at a mean of 7.6 years after vaccine injection. The mean GMCs in serotypes $6 \mathrm{~B}, 14,19 \mathrm{~F}$, and $23 \mathrm{~F}$ after PPV and PCV vaccination ranged from 2 to $9 \mu \mathrm{g} / \mathrm{ml}$, which is comparable to our result.

Studies on the efficacy of pneumococcal vaccine in patients with heterotaxy are limited. Mikoluc et al (26) analyzed 30 asplenic patients and found that the GMCs were low after PPV immunization. The PCV booster induced a satisfactory immune response in asplenic patients. Smets et al (27) analyzed the pneumococcal vaccine booster response in nine patients with anatomical asplenism and 10 patients with functional asplenism. In their study, 6 years after PPV vaccine 


\section{Articles | shao et al.}

injection, the baseline GMC was higher than $1 \mu \mathrm{g} / \mathrm{ml}$ in all serotypes, except in serotypes 4 and 18C. The booster response was good for both PCV7 and PPV in all serotypes, except in serotype 23F, which showed better response for PCV7. Wasserstrom et al (28) studied the pneumococcal antibody response after PPV injection in 26 splenectomized patients and reported that the antibody response in patients with spherocytosis receiving splenectomy was similar to that of the control group. However, in autoimmune patients receiving splenectomy, the antibody response was significantly lower than that of the control group. The authors suggested that because patients with autoimmunity had splenectomy at a significantly older age than did those with spherocytosis, age-related loss of extra-splenic sites necessary for the maintenance or function of memory B cells might lead to impaired immunity in autoimmune patients. Nived et al (17) and Stanford et al (29) studied adult splenectomy patients and found adequate GMC levels more than 5 years after PPV immunization. The immune response was found to be strong after taking PCV boosters.

The present study, which used a large cohort with 42 patients with heterotaxy, revealed that the GMCs of the four serotypes in patients with heterotaxy are comparable to patients with CCHD after PCV or PPV vaccination. The GMC is higher than the protective level in most of these patients, even in those with low IgM memory B-cell percentages and in those without spleen. This result suggests that both PPV and PCV are effective in these high-risk patients, despite an impaired splenic immune function. However, four patients, two in the heterotaxy group but with normal splenic function and the other two in the CCHD group, had poor response to PCV7, especially for serotype 23F. Two other patients had low GMC after PPV injection: one each in serotype $23 \mathrm{~F}$ and $6 \mathrm{~B}$. All patients had long durations between the last vaccination and antibody check. In our previous study in normal children, we found that pneumococcal antibody titers decreased with time and increased significantly after the booster dose (30). These results suggest that PPV (for those who had received prior PCV) or PCV13 (for those who had received prior PPV) booster is necessary to enhance the antibody titer in certain serotypes in some patients.

\section{Study Limitations}

As a very rare disease, it is difficult to enroll a large number of patients. It is also difficult to prospectively evaluate the durability of pneumococcal antibody response in this rare disease. However, we still found a good antibody response to pneumococcal vaccine in these heterotaxy patients.

\section{CONCLUSION}

The adherence to the pneumococcal vaccination guidelines in patients with heterotaxy was poor, and most of these patients did not receive additional PPV or new PCV boosters. Further education regarding the new guidelines is necessary for health-care professionals to prevent severe sepsis in the high- risk patients. The antibody response is adequate for both PPV and PCVs but may decrease with time in certain serotypes in some patients. Additional booster doses with PPV or PCV13 should be considered to enhance the protection effect.

\section{ACKNOWLEDGMENTS}

The authors thank Professor Sui-Yuan Chang and her laboratory for their contribution to the study.

\section{STATEMENT OF FINANCIAL SUPPORT}

This study was supported by National Science Council from government in Taiwan (grant 101-2314-B-002-034-MY3)

Disclosure: The authors declare no conflict of interest.

\section{REFERENCES}

1. Park MK Cyanotic congenital heart defect: heterotaxia (atrial isomerism, splenic syndromes). In: Park MK, ed. Pediatric Cardiology for Practitioners. St. Louis, Mosby, 2008.

2. Anagnostopoulos PV, Pearl JM, Octave C, et al. Improved current era outcomes in patients with heterotaxy syndromes. Eur J Cardiothorac Surg 2009;35:871-7 (discussion 877-8).

3. Wu MH, Wang JK, Lue HC. Sudden death in patients with right isomerism (asplenism) after palliation. J Pediatr 2002;140:93-6.

4. Prendiville TW, Barton LL, Thompson WR, Fink DL, Holmes KW. Heterotaxy syndrome: defining contemporary disease trends. Pediatr Cardiol 2010;31:1052-8.

5. Chiu SN, Shao PL, Wang JK, et al. Severe bacterial infection in patients with heterotaxy syndrome. J Pediatr 2014;164:99-104.e1.

6. William BM, Thawani N, Sae-Tia S, Corazza GR. Hyposplenism: a comprehensive review. Part II: clinical manifestations, diagnosis, and management. Hematology 2007;12:89-98.

7. Spelman D, Buttery J, Daley A, et al. Guidelines for the prevention of sepsis in asplenic and hyposplenic patients. Intern Med J 2008;38: 349-56.

8. Davies JM, Lewis MP, Wimperis J, Rafi I, Ladhani S, Bolton-Maggs PH. British Committee for Standards in $\mathrm{H}$ Review of guidelines for the prevention and treatment of infection in patients with an absent or dysfunctional spleen: prepared on behalf of the British Committee for Standards in Haematology by a working party of the Haemato-Oncology task force. Br J Haematol 2011;155:308-17.

9. Alter SJ. Pneumococcal infections. Pediatr Rev 2009;30:155-64 (quiz 164).

10. Tan TQ. Pediatric invasive pneumococcal disease in the United States in the era of pneumococcal conjugate vaccines. Clin Microbiol Rev 2012;25: 409-19.

11. Schutze GE, Mason EO Jr., Barson WJ, et al. Invasive pneumococcal infections in children with asplenia. Pediatr Infect Dis J 2002;21: $278-82$.

12. Wei SH, Chiang CS, Chen CL, Chiu CH. Pneumococcal disease and use of pneumococcal vaccines in Taiwan. Clin Exp Vaccine Res 2015;4:121-9.

13. Bennett NM, Whitney CG, Moore M, Pilishvili T, Dooling KL. Use of 13valent pneumococcal conjugate vaccine and 23-valent pneumococcal polysaccharide vaccine for adults with immunocompromising conditions: recommendations of the Advisory Committee on Immunization Practices (ACIP) (Reprinted from MMWR vol 40, pg 816, 2012). JAMA 2013;309: 334-6.

14. Lu CL, Chang SY, Chuang YC, et al. Revaccination with 7-valent pneumococcal conjugate vaccine elicits better serologic response than 23valent pneumococcal polysaccharide vaccine in HIV-infected adult patients who have undergone primary vaccination with 23-valent pneumococcal polysaccharide vaccine in the era of combination antiretroviral therapy. Vaccine 2014;32:1031-5.

15. Kruetzmann S, Rosado MM, Weber H, et al. Human immunoglobulin $M$ memory B cells controlling Streptococcus pneumoniae infections are generated in the spleen. J Exp Med 2003;197:939-45. 
16. Chiu SN, Shao PL, Wang JK, et al. Low immunoglobulin M memory B-cell percentage in patients with heterotaxy syndrome correlates with the risk of severe bacterial infection. Pediatr Res 2015;79:271-7.

17. Nived P, Jorgensen CS, Settergren B. Vaccination status and immune response to 13-valent pneumococcal conjugate vaccine in asplenic individuals. Vaccine 2015;33:1688-94.

18. WHO. Recommendations for the productionand control of pneumococcal conjugate vaccines. World Health Tech Rep Ser 2005;927:64-98.

19. Ochoa-Gondar O, Vila-Corcoles A, Rodriguez-Blanco T, et al. Effectiveness of the 23-valent pneumococcal polysaccharide vaccine against community-acquired pneumonia in the general population aged $>=60$ years: 3 years of follow-up in the CAPAMIS Study. Clin. Infect. Dis. 2014;58:909-17.

20. Feldman C, Anderson R. Review: current and new generation pneumococcal vaccines. J Infect 2014;69:309-25.

21. Pittet LF, Posfay-Barbe KM. Pneumococcal vaccines for children: a global public health priority. Clin Microbiol Infect 2012;18 (Suppl 5): 25-36.

22. Pilishvili T, Lexau C, Farley MM, et al. Surveillance ABC. Sustained reductions in invasive pneumococcal disease in the era of conjugate vaccine. J Infect Dis 2010;201:32-41.

23. Hsieh YC, Lin PY, Chiu CH, et al. National survey of invasive pneumococcal diseases in Taiwan under partial PCV7 vaccination in 2007: emergence of serotype 19 A with high invasive potential. Vaccine 2009;27:5513-8.25
24. Hsieh YC, Huang YC, Lin HC, et al. Characterization of invasive isolates of Streptococcus pneumoniae among Taiwanese children. Clin Microbiol Infect 2009;15:991-6.

25. Sigurdardottir ST, Center KJ, Davidsdottir K, Arason VA, Hjalmarsson B, Elisdottir R, Ingolfsdottir G, Northington R, Scott DA, Jonsdottir I 2014 Decreased immune response to pneumococcal conjugate vaccine after 23-valent pneumococcal polysaccharide vaccine in children. Vaccine 32:417-424.

26. Mikoluc B, Kayhty H, Bernatowska E, Motkowski R. Immune response to the 7-valent pneumococcal conjugate vaccine in 30 asplenic children. Eur J Clin Microbiol Infect Dis 2008;27:923-8.

27. Smets F, Bourgois A, Vermylen C, et al. Randomised revaccination with pneumococcal polysaccharide or conjugate vaccine in asplenic children previously vaccinated with polysaccharide vaccine. Vaccine 2007;25: 5278-82.

28. Wasserstrom H, Bussel J, Lim LC, Cunningham-Rundles C. Memory B cells and pneumococcal antibody after splenectomy. J Immunol 2008;181: 3684-9.

29. Stanford E, Print F, Falconer M, et al. Immune response to pneumococcal conjugate vaccination in asplenic individuals. Hum Vaccin 2009;5:85-91.

30. Shao PL, Lu CY, Chang LY, et al. Safety and immunogenicity of heptavalent pneumococcal conjugate vaccine booster in Taiwanese toddlers. J Formos Med Assoc 2006;105:542-9. 